\title{
Clinical Significance of Lymph Node Ratio in Stage III Colorectal Cancer
}

\author{
Yo Han Park, Jae Im Lee, Jong Kyung Park, Hang Ju Jo, Won Kyung Kang, Chang Hyeok An \\ Department of Surgery, Uijeongbu St. Mary's Hospital, The Catholic University of Korea School of Medicine, Uijeongbu, Korea
}

Purpose: Recent literature has shown that lymph node ratio is superior to the absolute number of metastatic lymph nodes in predicting the prognosis in several malignances other than colorectal cancer. The aim of this study was to evaluate the prognostic significance of the lymph node ratio (LNR) in patients with stage III colorectal cancer.

Methods: We included 186 stage III colorectal cancer patients who underwent a curative resection over a 10-year period in one hospital. The cutoff point of LNR was chosen as 0.07 because there was significant survival difference at that LNR. The Kaplan-Meier and the Cox proportional hazard models were used to evaluate the prognostic effect according to LNR. Results: There was statistically significant longer overall survival in the group of LNR $>0.07$ than in the group of LNR $\leq 7$ $(\mathrm{P}=0.008)$. Especially, there was a survival difference for the N1 patients group $(\mathrm{LN}<4)$ according to LNR $(5$-year survival of N1 patients was lower in the group of LNR $>0.07, \mathrm{P}=0.025)$, but there was no survival difference for the N2 group (4 $\geq \mathrm{LN}$ ) according to LNR. The multivariate analysis showed that the LNR is an independent prognostic factor.

Conclusions: LNR can be considered as a more accurate and potent modality for prognostic stratifications in patients with stage III colorectal cancer.

Keywords: Colorectal cancer; Metastatic lymph node ratio; Prognostic factor

\section{INTRODUCTION}

Colorectal cancer has the second highest incidence globally among malignant tumors, and the prevalence and the incidence rate in South Korea are rising. Earlier studies have shown that the prognostic factors for colorectal cancer are early detection, age at diagnosis, location of tumor, histological differentiation, depth of invasion, lymph node metastasis, preoperative and postoperative plasma carcino embryonic antigen level, and degree of venous and neural invasion. Of these factors, depth of invasion, lymph node metastasis and metastasis to other organs [1], as reflected in the current TNM staging (T, depth of invasion; N, lymph node metastasis; $M$,

Received: May 20, 2011 Accepted: August 7, 2011

Correspondence to: Chang Hyeok An, M.D.

Department of Surgery, Uijeongbu St.Mary's Hospital, The Catholic University of Korea School of Medicine, 65-1 Geumo-dong, Uijeongbu 480-717, Korea

Tel: +82-31-820-3998, Fax: +82-31-847-2717

E-mail: achcolo@catholic.ac.kr

\section{(c) 2011 The Korean Society of Coloproctology}

This is an open-access article distributed under the terms of the Creative Commons Attribution NonCommercial License (http://creativecommons.org/licenses/by-nc/3.0) which permits unrestricted noncommercial use, distribution, and reproduction in any medium, provided the original work is properly cited. distant metastasis), form the basis for determining the prognosis [2]. Among them, lymph node metastasis is known to be one of the most important factors. As the survival rate has been shown to increase after adjuvant chemotherapy [2,3], the existence of lymph node metastasis is grounds for advocating postoperative adjuvant chemotherapy or radiotherapy. Therefore, reporting the status of lymph nodes (both the number of nodes harvested and the number of metastatic lymph nodes) accurately is very critical in deciding the postoperative treatments and in giving a prognosis. However, the expected prognosis when determined by the number of metastatic lymph nodes is not consistent in the patients whose stage is same. In addition, it is difficult to determine lymph node metastasis objectively as lymph node metastasis depends on the individual characteristics of the patient, the competence of the surgeon and the ability of the pathologist to isolate and collect lymph nodes $[4,5]$. Current studies have found that the number of harvested lymph nodes is related to the disease-free and the overall survival rates of colorectal cancer patients [6-8]. In other words, a higher harvested lymph node number is associated with a higher survival rate in patients treated with a radical tumor resection $[9,10]$.

Against this background, a metastatic lymph node ratio (LNR) rather than an absolute metastatic lymph node count has been re- 
ported to be meaningful for reducing stage migration and for giving a prognosis for other types of solid cancers (stomach [11-13], breast [14], bladder [15], pancreas [16] and lung cancer [17]). Studies on the clinical meaning of the LNR as a prognostic factor have been performed for colorectal cancer patients [16, 18-23]. However, the clinical meaning of the LNR in colorectal cancer patients is still controversial as some researchers found no correlation between the LNR and the survival rate while others insisted that the LNR was a more critical prognostic factor than simple lymph node metastasis. Therefore, we conducted this study to determine whether the LNR is a stronger prognostic factor than the absolute number of metastatic lymph nodes in patients with stage III colorectal cancer.

\section{METHODS}

One hundred eighty-six stage III colorectal cancer patients undergoing a radical resection over 10 years at our hospital were evaluated. Patients with a histologically-diagnosed non-adenocarcinomas or with an appendiceal or anal cancer were excluded from this study.

Laparoscopic or open surgery was conducted for all of the patients by one surgeon, and depending on the location of the tumor, a standard colonic resection with lymph node dissection was done. The age and gender of the patients, the location of the tumors, the histological differentiation of the tumors, the depth of invasion, the number of harvested lymph nodes, the number of metastatic lymph nodes and the LNR were analyzed after the surgery. The LNR was defined as a ratio of the number of metastatic lymph nodes to the number of total harvested lymph nodes. The depth of invasion and the number of metastatic lymph nodes helped stage the disease using the current AJCC 7th edition [2].

The location of the tumors was classified into the right colon (from the cecum to the distal transverse colon), the left colon (from the splenic flexure to the sigmoid colon) and the rectum. Patients diagnosed with a stage III or higher disease postoperatively underwent adjuvant chemotherapy, and postoperative follow-up was performed through chest X-ray, abdominal computed tomography $(\mathrm{CT})$ and tumor markers at six-month intervals for the first two years and at one-year intervals for the next three years. Local recurrence or metastasis to other organs was confirmed histologically, if it was available, and with radiologic examination if the former was not available. The LNR was divided into three regions,

Table 1. Total harvested lymph node (LN) numbers and LN number when harvested $\mathrm{LN} \geq 12$ according to tumor location

\begin{tabular}{lcr}
\hline & \multicolumn{2}{c}{ Total harvested / harvested $\mathrm{LN} \geq 12$} \\
\cline { 2 - 3 } & Patient $\mathrm{no}$. & \multicolumn{1}{c}{ Mean \pm standard deviation } \\
\hline Right & $117 / 83$ & $18.97 \pm 9.7 / 22.13 \pm 8.15$ \\
Left & $183 / 89$ & $14.92 \pm 9.53 / 20.33 \pm 7.17$ \\
Rectum & $184 / 86$ & $13.26 \pm 10.23 / 21.19 \pm 9.83$ \\
Total & $484 / 258$ & $15.27 \pm 10.07 / 21.20 \pm 8.44$ \\
\hline
\end{tabular}

less than $0.07,0.08-0.20$, and more than 0.20 , as suggested by Peschaud et al. [20], but the survival rates of the groups with LNR of $0.08-0.20$ and over 0.20 were not considerably different, so the patients were finally divided into two groups: One with LNR of less than 0.07 and the other with a LNR of 0.07 or greater.

The statistical analysis was conducted with SPSS ver. 12.0 (SPSS Inc., Chicago, IL, USA), and the survival curve was prepared using a Kaplan-Meier analysis. The univariate analysis and the multivariate analysis of prognostic factors were performed using the log-rank method and the Cox proportional hazard ratio, respectively. A P-value of less than 0.05 was considered to be statistically significant.

Table 2. Clinicopathological characteristics of stage III colorectal cancer patients and 5-year survival (univariate analysis)

\begin{tabular}{|c|c|c|c|}
\hline Characteristics & $\begin{array}{c}\text { No. of } \\
\text { cases (\%) }\end{array}$ & $\begin{array}{c}\text { 5-year } \\
\text { survival (\%) }\end{array}$ & P-value \\
\hline \multicolumn{4}{|l|}{ Age } \\
\hline$<60$ & $56(30.1)$ & 79 & NS \\
\hline$\geq 60$ & $130(69.9)$ & 73 & \\
\hline \multicolumn{4}{|l|}{ Sex } \\
\hline Male & $110(59.1)$ & 74 & NS \\
\hline Female & 76 (40.9) & 75 & \\
\hline \multicolumn{4}{|l|}{ T stage } \\
\hline $\mathrm{T} 1$ & $7(3.8)$ & 75 & 0.005 \\
\hline T2 & $16(8.6)$ & 75 & \\
\hline T3 & $129(69.4)$ & 64 & \\
\hline $\mathrm{T} 4$ & 34 (18.3) & 41 & \\
\hline \multicolumn{4}{|l|}{ N Stage } \\
\hline N1 & $125(67.2)$ & 67 & 0.006 \\
\hline N2 & $61(32.8)$ & 49 & \\
\hline \multicolumn{4}{|l|}{ TNM stage } \\
\hline$\| \mathrm{II}$ & 21 (11.3) & 88 & 0.014 \\
\hline$\| I B$ & $104(55.9)$ & 62 & \\
\hline IIIC & $61(32.8)$ & 49 & \\
\hline \multicolumn{4}{|c|}{ Lymphatic invasion } \\
\hline No & 25 (13.6) & 67 & NS \\
\hline Yes & $159(86.4)$ & 60 & \\
\hline \multicolumn{4}{|l|}{ Venous invasion } \\
\hline No & 171 (93.4) & 62 & 0.006 \\
\hline Yes & $12(6.6)$ & 39 & \\
\hline \multicolumn{4}{|c|}{ Perineural invasion } \\
\hline No & 132 (72.9) & 65 & 0.020 \\
\hline Yes & $49(27.1)$ & 45 & \\
\hline
\end{tabular}

NS, no significant. 


\section{RESULTS}

Four hundred eighty-four colorectal cancer patients underwent radical surgery at our hospital over 10 years. The tumors were located in the right colon, the left colon and the rectum in 117, 183, and 184 patients, respectively. When considering the overall resections (both adequate and inadequate as per the AJCC criteria), the total numbers of harvested lymph nodes were 18, 14 and 13, on average, for the right colon, the left colon and the rectum, respectively; the harvested lymph node number for the right colon was significantly higher $(\mathrm{P}<0.001)$ in this group. However, when exclusively considering cases with adequate resections with 12 or more LNs, the numbers of lymph nodes harvested by the locations of

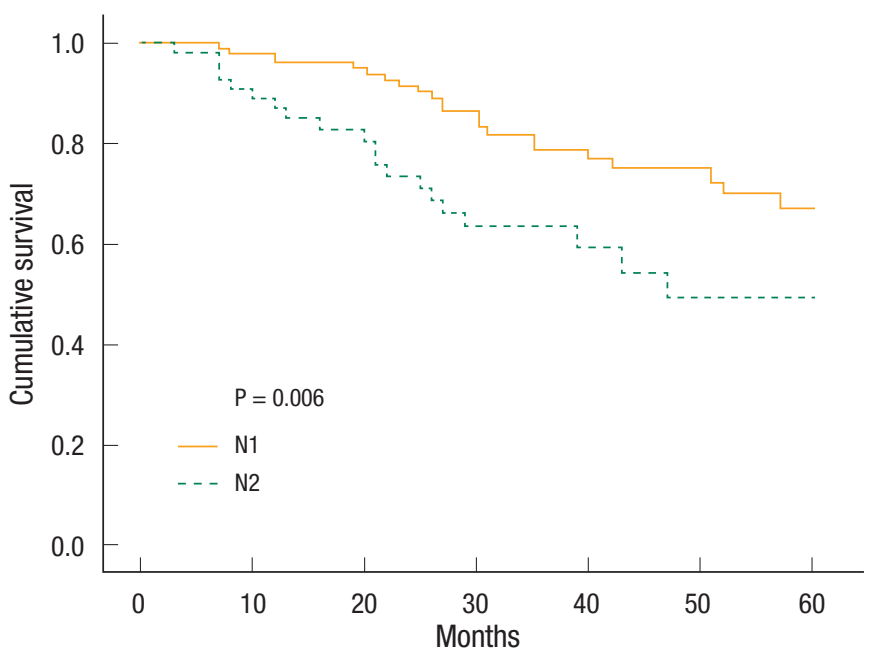

Fig. 1. Five-year survival curve in stage III colorectal cancer patients according to metastatic lymph node number ( $\mathrm{N}$ staging).

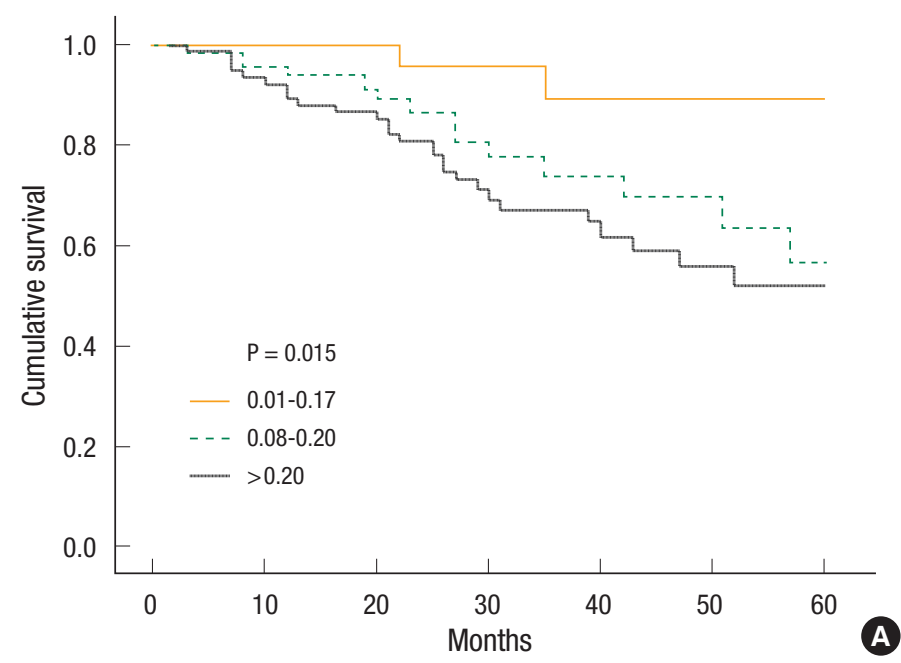

the tumors were not significantly different (Table 1).

This study included only 186 stage III colorectal cancer patients, and patients with ages over 60 years (130) accounted for $69.9 \%$ of the total. The clinicopathological characteristics of the patients are shown in Table 2. Among these patients, the five-year survival rate was lower for a higher T stage and for more advanced stage III colorectal cancer. The survival rate of the stage $\mathrm{N} 1$ group was higher than that of the stage N2 group, $67 \%$ and $49 \%$ respectively (Fig. 1).

Fig. $2 \mathrm{~A}$ presents the five-year survival rate by LNR in stage III colorectal cancer patients. The five-year survival rate was the highest in the group with a LNR of less than 0.07 while those of the groups with a LNR of $0.08-0.20$ and more than 0.20 were not significantly different. When the total patients were finally divided into two groups with a LNR of less than 0.07 and a LNR of 0.07 or greater, the former recorded a higher five-year survival rate, and the difference was statistically significant (Fig. 2B).

To determine the effect of LNR on the prognosis, the five-year survival rate by LNR was investigated at each $\mathrm{N}$ stage, and the survival curves are shown in Fig. 3A and Fig. 3B. In the N1 group with three or less metastatic lymph nodes, the survival rate of the group with a LNR of less than 0.07 was significantly higher than that of the group with a LNR of 0.07 or greater. In the N2 group, the difference in the survival rates between the two groups was not significant.

When colon cancer and rectal cancer were compared, the fiveyear survival rates of colon cancer patients were different with a LNR of 0.07 as the standard, but the difference did not have a statistical meaning $(\mathrm{P}=0.143)$ (Fig. $4 \mathrm{~A})$. The rates of rectal cancer patients showed a statistically significant difference with the same standard (Fig. 4B).

A multivariate analysis of the clinicopathological characteristics, which were found to be significant with a P-value of less than 0.05

Fig. 2. (A) Five-year survival curve in stage III colorectal cancer patients according to metastatic lymph node ratio ( 3 groups: LNR, 0.01-0.07; $0.08-0.20 ;>0.20)$. (B) Five-year survival curve in stage III colorectal cancer patients according to metastatic lymph node ratio (2 groups: LNR, $0.01-0.07 ;>0.07)$. 

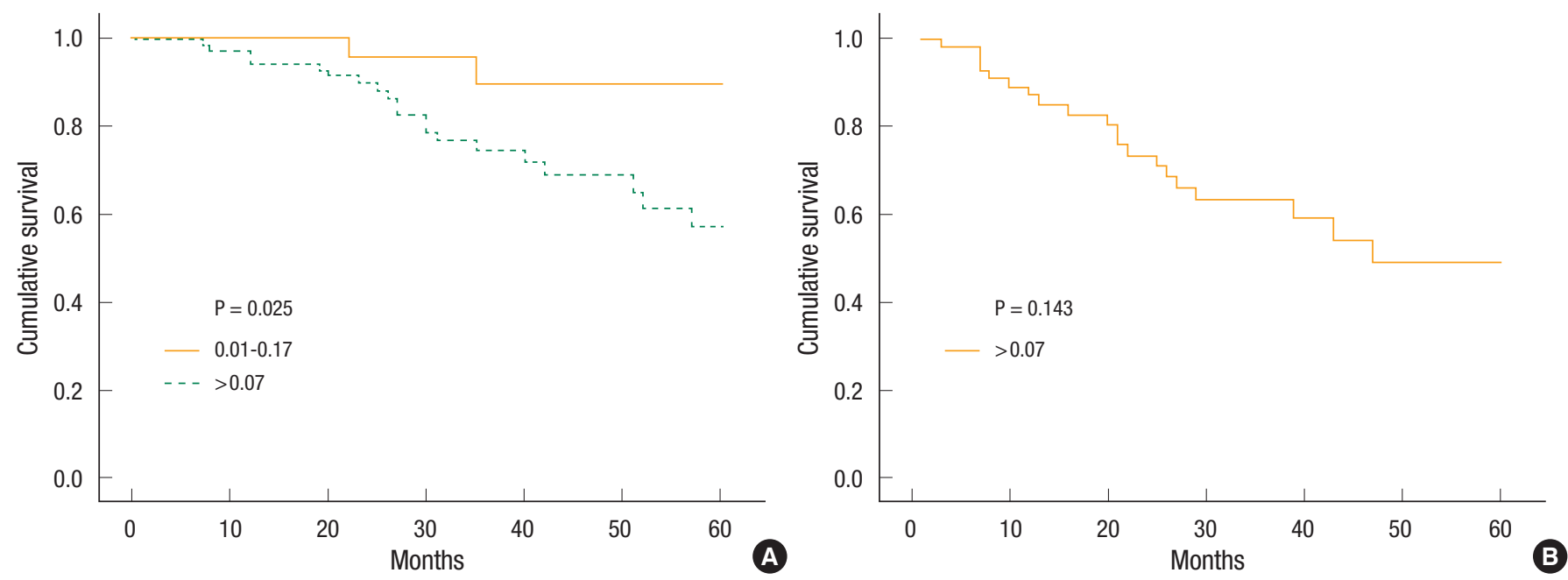

Fig. 3. (A) Five-year survival curve in metastatic lymph node number $<4$ (N1 staging) cancer patients according to metastatic lymph node ratio. (B) Five-year survival curve in metastatic lymph node number $\geq 4$ (N2 staging) cancer patients according to metastatic lymph node ratio.
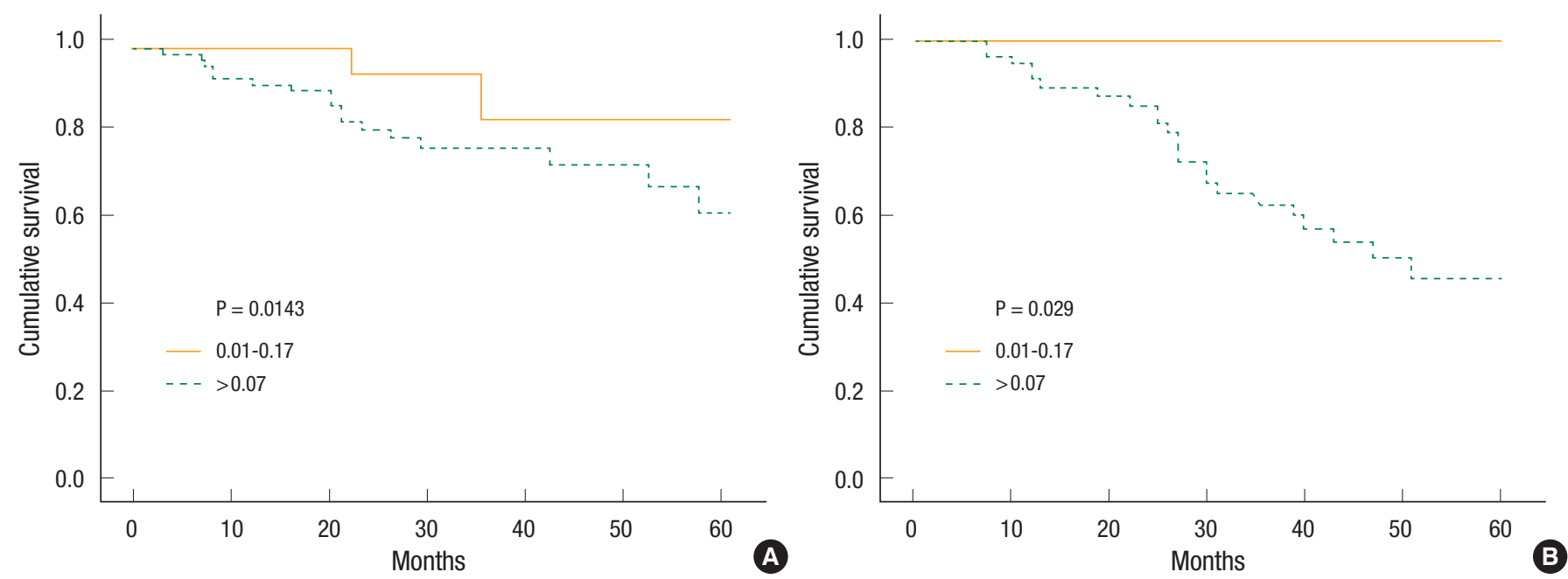

Fig. 4. (A) Five-year survival curve in stage III colon cancer patients according to metastatic lymph node ratio (cut-off point, 0.07). (B) Fiveyear survival curve in stage III rectal cancer patients according to metastatic lymph node ratio (cut-off point, 0.07).

Table 3. Significance of prognostic factors through multivariate analysis

\begin{tabular}{lccc}
\hline Characteristic & Odds ratio & $95 \% \mathrm{Cl}$ & P-value \\
\hline Lymph node ratio & 4.377 & $1.029-18.607$ & 0.046 \\
Stage & 1.694 & $1.001-2.869$ & 0.048 \\
Per neural invasion & 1.004 & $1.004-3.451$ & 0.050 \\
\hline
\end{tabular}

$\mathrm{Cl}$, confidence interval.

on the univariate analysis, of stage III colorectal cancer patients revealed that LNR, T staging and neural invasion had a significant correlation with the prognosis. Of them, LNR was found to be one of the most critical prognostic factors, with an odds ratio of 4.3 (Table 3).

\section{DISCUSSION}

Amongst the prognostic factors, the depth of invasion, lymph node metastasis and metastasis to other organs have been found to be closely related with the prognosis for colorectal cancer [1]. The status of lymph node metastasis has been recognized as the one factor exerting the strongest influence on the prognosis. However, the lymph node metastasis depends on the individual characteristics of the patient and the competence of the surgeon and the pathologist, thus making it difficult to assess the status accurately $[4,5]$. For example, more lymph nodes are harvested in young patients, females and thin patients when considering the demographic characteristics, from the right colon when considering the location of the tumor and at larger hospitals and by more experienced surgeons 
in general $[4,6,24,25]$. In addition, lymph node metastasis is found through RT-PCR in patients without metastasis in the current TNM staging [26], and stage migration (reported first by Feinstein and called Will Rogers phenomenon; the current AJCC TNM staging says that the same number of metastatic lymph nodes leads to the same prognosis even though the numbers of harvested lymph nodes are different [27]) can be shown, even in stage III colorectal cancer patients, to cause a deviation in the survival rate when only the absolute number of metastatic lymph nodes is considered [18]. Because giving a prognosis for colorectal cancer based on the above criteria has led to some criticism, the concept of the LNR, which has been accepted as a clinically meaningful prognostic factor for other types of solid malignant tumors, has been introduced in studies on colorectal cancer [19-23, 28].

According to the results of this study, when the patients in $\mathrm{N} 1$ and N2 stages were divided by LNR, respectively, the five-year survival rates were different by LNR in patients in the N1 stage (Fig. 3A). That shows that the patients divided by $\mathrm{N}$ staging based on the absolute number of metastatic lymph nodes are not distributed consistently and that the use of LNR, along with the current TNM staging, can give a better prognosis. Unlike our expectation, the fiveyear survival rates of the patients in N2 stage were not different by LNR (Fig. 3B) and that is thought to have been due to cut off of 0.07 not being appropriate for showing a significant difference in the survival rates among the patients in N2 stage.

This study compared the survival rate by LNR after dividing the colorectal cancer patients into colon cancer and rectal cancer patients. When the LNR (0.07) used for rectal cancer was applied to colon cancer, the accuracy of predicting the survival rate was slightly reduced (Fig. 4). A cut-off point to present a statistically significant difference in survival rate of colon cancer patients must be different from the value of 0.07 used for rectal cancer; thus, appropriate the cut-off points need to be determined to obtain a significant difference in the survival rate by the location of the tumor.

The multivariate analysis found that the LNR, independently, had the most important effect among the prognostic factors for colorectal cancer (Table 3) because the LNR is thought to decrease the effect of stage migration in colorectal cancer. Inoue et al. [12] and Bando et al. [13] insisted that the LNR was meaningful for giving a prognosis because it reduced stage migration. These findings suggest that the LNR can replace $\mathrm{N}$ staging and that a revision of the current TNM staging is needed.

Previous studies have used various cut-off points for the LNR: 0.0, $0.05,0.10,0.20,0.25,0.40,0.50$, and 0.75 . Most of the studies determined the cut-off points by using the LNR to draw the KaplanMeier survival curve [29]. This study also performed statistical analyses with the various cut-off points of the LNR applied in previous studies and found that 0.07 and 0.20 , as suggested by Peschaud et al. [20], produced the largest difference in the survival rate when all patients were divided into three groups with the cut-off points. In each study, including this study, the survival rate, the diseasefree rate and cancer-related survival rate were significantly differ- ent among the patients classified by cut-off points. However, the studies utilized various cut-off points. Thus, if the LNR is to be used as a prognostic factor, a standardization of cut-off points for the LNR is necessary for stratification of stages with better correlation with the prognosis.

This study had a limitation in that the number of total subjects was not as large as in a retrospective study. In addition, it included all rectal cancer patients for whom lymph node resection had been limited due to preoperative radiotherapy.

Our data show that patients divided by $\mathrm{N}$ staging based on an absolute number of metastatic lymph nodes are not distributed consistently. The use of LNR, along with the current TNM staging, can better predict the prognosis for N1 stage of colorectal cancer and may be an independent prognostic factor in stage III colorectal cancer. A large-scale study to determine appropriate cut-off points for LNR to play a role as an independent prognostic factor in stage III colorectal cancer is needed.

\section{CONFLICT OF INTEREST}

No potential conflict of interest relevant to this article was reported.

\section{REFERENCES}

1. Astler VB, Coller FA. The prognostic significance of direct extension of carcinoma of the colon and rectum. Ann Surg 1954;139: 846-52.

2. Greene FL; American Joint Committee on Cancer; American Cancer Society. AJCC cancer staging manual. 6th ed. New York: Springer-Verlag; 2002.

3. Van Cutsem EJ, Oliveira J; ESMO Guidelines Working Group. Colon cancer: ESMO clinical recommendations for diagnosis, adjuvant treatment and follow-up. Ann Oncol 2008;19 Suppl 2:ii29-30.

4. Wong SL, Ji H, Hollenbeck BK, Morris AM, Baser O, Birkmeyer JD. Hospital lymph node examination rates and survival after resection for colon cancer. JAMA 2007;298:2149-54.

5. Evans MD, Barton K, Rees A, Stamatakis JD, Karandikar SS. The impact of surgeon and pathologist on lymph node retrieval in colorectal cancer and its impact on survival for patients with Dukes' stage B disease. Colorectal Dis 2008;10:157-64.

6. Vather R, Sammour T, Kahokehr A, Connolly AB, Hill AG. Lymph node evaluation and long-term survival in Stage II and Stage III colon cancer: a national study. Ann Surg Oncol 2009;16:585-93.

7. Le Voyer TE, Sigurdson ER, Hanlon AL, Mayer RJ, Macdonald JS, Catalano PJ, et al. Colon cancer survival is associated with increasing number of lymph nodes analyzed: a secondary survey of intergroup trial INT-0089. J Clin Oncol 2003;21:2912-9.

8. Baxter NN, Virnig DJ, Rothenberger DA, Morris AM, Jessurun J, Virnig BA. Lymph node evaluation in colorectal cancer patients: a population-based study. J Natl Cancer Inst 2005;97:219-25.

9. Johnson PM, Porter GA, Ricciardi R, Baxter NN. Increasing negative lymph node count is independently associated with improved 
long-term survival in stage IIIB and IIIC colon cancer. J Clin Oncol 2006;24:3570-5.

10. Chang GJ, Rodriguez-Bigas MA, Skibber JM, Moyer VA. Lymph node evaluation and survival after curative resection of colon cancer: systematic review. J Natl Cancer Inst 2007;99:433-41.

11. Persiani R, Rausei S, Biondi A, Boccia S, Cananzi F, D’Ugo D. Ratio of metastatic lymph nodes: impact on staging and survival of gastric cancer. Eur J Surg Oncol 2008;34:519-24.

12. Inoue K, Nakane Y, Iiyama H, Sato M, Kanbara T, Nakai K, et al. The superiority of ratio-based lymph node staging in gastric carcinoma. Ann Surg Oncol 2002;9:27-34.

13. Bando E, Yonemura Y, Taniguchi K, Fushida S, Fujimura T, Miwa $\mathrm{K}$. Outcome of ratio of lymph node metastasis in gastric carcinoma. Ann Surg Oncol 2002;9:775-84.

14. Weir L, Speers C, D'yachkova Y, Olivotto IA. Prognostic significance of the number of axillary lymph nodes removed in patients with node-negative breast cancer. J Clin Oncol 2002;20:1793-9.

15. Herr HW, Bochner BH, Dalbagni G, Donat SM, Reuter VE, Bajorin DF. Impact of the number of lymph nodes retrieved on outcome in patients with muscle invasive bladder cancer. J Urol 2002; 167:1295-8.

16. Berger AC, Watson JC, Ross EA, Hoffman JP. The metastatic/examined lymph node ratio is an important prognostic factor after pancreaticoduodenectomy for pancreatic adenocarcinoma. Am Surg 2004;70:235-40.

17. Gajra A, Newman N, Gamble GP, Kohman LJ, Graziano SL. Effect of number of lymph nodes sampled on outcome in patients with stage I non-small-cell lung cancer. J Clin Oncol 2003;21:1029-34.

18. Wang J, Hassett JM, Dayton MT, Kulaylat MN. Lymph node ratio: role in the staging of node-positive colon cancer. Ann Surg Oncol 2008;15:1600-8.

19. Schumacher P, Dineen S, Barnett C Jr, Fleming J, Anthony T. The metastatic lymph node ratio predicts survival in colon cancer. Am J Surg 2007;194:827-31.

20. Peschaud F, Benoist S, Julie C, Beauchet A, Penna C, Rougier P, et al. The ratio of metastatic to examined lymph nodes is a powerful independent prognostic factor in rectal cancer. Ann Surg 2008; 248:1067-73.

21. Lee HY, Choi HJ, Park KJ, Shin JS, Kwon HC, Roh MS, et al. Prognostic significance of metastatic lymph node ratio in node-positive colon carcinoma. Ann Surg Oncol 2007;14:1712-7.

22. Derwinger K, Carlsson G, Gustavsson B. A study of lymph node ratio as a prognostic marker in colon cancer. Eur J Surg Oncol 2008;34:771-5.

23. Chin CC, Wang JY, Yeh CY, Kuo YH, Huang WS, Yeh CH. Metastatic lymph node ratio is a more precise predictor of prognosis than number of lymph node metastases in stage III colon cancer. Int J Colorectal Dis 2009;24:1297-302.

24. Rosenberg R, Friederichs J, Schuster T, Gertler R, Maak M, Becker $\mathrm{K}$, et al. Prognosis of patients with colorectal cancer is associated with lymph node ratio: a single-center analysis of 3,026 patients over a 25-year time period. Ann Surg 2008;248:968-78.

25. Kelder W, Inberg B, Plukker JT, Groen H, Baas PC, Tiebosch AT. Effect of modified Davidson's fixative on examined number of lymph nodes and TNM-stage in colon carcinoma. Eur J Surg Oncol 2008;34:525-30.

26. Telian SH, Bilchik AJ. Significance of the lymph node ratio in stage III colon cancer. Ann Surg Oncol 2008;15:1557-8.

27. Feinstein AR, Sosin DM, Wells CK. The Will Rogers phenomenon: stage migration and new diagnostic techniques as a source of misleading statistics for survival in cancer. N Engl J Med 1985; 312:1604-8.

28. Berger AC, Sigurdson ER, LeVoyer T, Hanlon A, Mayer RJ, Macdonald JS, et al. Colon cancer survival is associated with decreasing ratio of metastatic to examined lymph nodes. J Clin Oncol 2005;23:8706-12.

29. Ceelen W, Van Nieuwenhove Y, Pattyn P. Prognostic value of the lymph node ratio in stage III colorectal cancer: a systematic review. Ann Surg Oncol 2010;17:2847-55. 